\title{
Respuestas
}

Año 17

No. 1

Junio 2012

ISSN 0122-820X

\section{Desarrollo e implementación de un módulo didáctico de automatización bajo una red de comunicación industrial Modbus}

\author{
Moreno G. Francisco E. ${ }^{1} \mid$ Becerra V. José A. ${ }^{2} \mid$ Mondragón E. Jorge E. $^{3}$
}

Recibido:

Diciembre 12 de 2011

Aceptado:

Mayo 18 de 2012
20

\footnotetext{
${ }^{1}$ Doctor en Ingeniería Mecánica. femgarcia@ufps.edu.co, Grupo de Investigación en Automatización y Control - GIAC, Universidad Francisco de Paula Santander, Avenida Gran Colombia No. 12E-96B Colsag. Cúcuta Colombia.

${ }^{2}$ Magister en controles industriales. josearmandobv@ufps.edu. co, Grupo de Investigación en Automatización y Control GIAC, Universidad Francisco de Paula Santander, Avenida Gran Colombia No. 12E-96B Colsag. Cúcuta - Colombia.

${ }^{3}$ Ingeniero Electrónico, jorgeenriqueme@ufps.edu. co, Grupo de Investigación en Automatización y Control GIAC, Universidad Francisco de Paula Santander, Avenida Gran Colombia No. 12E-96B Colsag. Cúcuta - Colombia.
}

\section{Resumen}

Este trabajo presenta el desarrollo y la implementación de un módulo didáctico bajo una red de comunicación con protocolo Modbus RTU, integrando elementos de automatización industrial como lo son una interfaz gráfica (HMI), dos controladores lógicos programables PLC's y un variador de frecuencia altivar 71. La red de comunicación industrial implementada, alcanzó una máxima transferencia de datos en el orden de $19200 \mathrm{kbit} / \mathrm{s}$, lo cual permitió desde un dispositivo maestro tener la capacidad de acceso, control y tratamiento a todos los registros internos de memoria de sus dispositivos esclavos. El desarrollo de este proyecto es un aporte a la solución de problemas relacionados a la transferencia de información entre diferentes dispositivos electrónicos y a la generación de alternativas de bajo costo para la adquisición y adaptación tecnológica en el área de comunicaciones industriales y automatización industrial Colombiana.

Palabras Clave: Protocolo Modbus, variador de frecuencia, PLC, HMI, automatización.

\section{Abstract}

This paper presents the development and implementation of a didactic module under an industrial communication with Modbus RTU protocol integrating elements of industrial automation as are graphical interface HMI, two programmable logic controllers PLC's and frequency inverter altivar 71.

The industrial communication network implemented, reached a maximum data transfer on the order of $19200 \mathrm{kbit} / \mathrm{s}$, which is allowed from a master device having the ability to access, control and treatment all internal registers of the memory slave devices. The development of this project is a contribution to solving problems related to the transfer of information between electronic devices and generating low-cost alternatives for the technology acquisition and adaptation in the field of industrial communications and industrial electronics Colombian.

Keywords: Modbus Protocol, frequency inverter, PLC, HMI, automation. 


\section{Introducción}

E aumento de los sistemas industriales automatizados ha obligado a la comunidad científica y académica a encontrar actualizaciones para todas las disciplinas dentro del campo de la automatización. Este hecho ha motivado a las universidades a disponer de laboratorios polivalentes que puedan ayudar a los estudiantes a desarrollarse y capacitarse en los diferentes campos de la ingeniería [1]. Hoy en día, la finalidad y el reto que se tiene en las universidades es ir a la vanguardia en innovación tecnológica, a partir de la creación de herramientas que faciliten el aprendizaje en la formación de profesionales más competitivos. Por lo tanto, el desarrollo de módulos didácticos contempla beneficios y perspectivas a ser alcanzados, permitiéndose al futuro profesional aprender y afrontar los diversos casos prácticos, que están presentes en la industria Colombiana.

El objetivo de este proyecto consistió en el diseño e implementación de un módulo didáctico conformado por una Human Machine Interface (HMI), dos controladores lógicos programables (PLC's) y un variador de frecuencia altivar 71 de la Schneider Electric, interconectados bajo un protocolo de comunicación industrial, en este caso MODBUS RTU. El desarrollo de este módulo permite a los estudiantes de Ingeniería Electrónica, Electromecánica y afines de la Universidad Francisco de Paula Santander (UFPS), contar con equipos de alta gama y afianzar conocimientos en las áreas de comunicación industrial, automatización, supervisión y control.

\section{Comunicaciones industriales en la automatización}

De acuerdo con la normatividad técnica, hay diferentes formas de reconocimiento para desarrollar proyectos de automatización industrial. Dos de estas formas son las normas de facto y las normas de jure [2] [3]. Un estándar de facto es, por definición, un producto que ha logrado el dominio a través de la aceptación del público y el uso. Por otro lado, un estándar de jure (por ejemplo el puerto físico RS - 485) que ha sido revisado por organizaciones como la Organización de Estandarización Internacional (ISO) y han sido legalmente aceptados. Los objetivos de estas organizaciones son la creación de al menos cuatro niveles de estandarización en la revisión: compatibilidad, intercambiabilidad, en común, y de referencia. Lo que estos cuatro niveles tratan de lograr, es la operacionalidad entre los productos y un acuerdo universal sobre su uso en la industria [4]. Es importante entender la distinción entre las dos normas anteriormente mencionadas cuando se empieza a ejecutar un proyecto donde los estándares de calidad deberán ser estrictamente utilizados.

Por lo tanto, el primer pasó para el desarrollo y organización de las comunicaciones consistió en decidir cómo se lograría la comunicación entre los dispositivos bajo las normas específicas de cada uno. En este caso, el protocolo Modbus sobre un medio físico RS-485 se visualizó como uno de los más aplicables debido a su amplio uso en la industria para llevar a cabo comunicaciones multipunto, así como su disponibilidad de la fuente abierta, que no requiere de regalías por el uso [4].

ElModbus es un protocolo de comunicación que ayuda al patrón de las comunicaciones multi-esclavo entre dispositivos electrónicos. Es una red de comunicación industrial, con capacidad para transferir datos entre un dispositivo maestro y hasta 247 dispositivos esclavos, cada uno con una dirección de esclavo única de 1 a 247, reportado en este artículo fue definido un dispositivo maestro y tres dispositivos esclavos, como se puede observar en la figura 1. 
En la figura 2 se presenta las capas de aplicación del protocolo Modbus según el modelo de interconexión de sistemas abiertos, open system interconnection (OSI) [5]. Con Modbus se permite la transferencia y almacenamiento de datos mediante el uso de bobinas y registros de memoria internos en cada dispositivos. Las bobinas se encargan de almacenar valores binarios simples mientras que los registros almacenan valores numéricos de 16 bits [6]. Tanto la bobina y los valores de registro se almacenan en tablas direccionadas específicamente con relación a los valores almacenados. Existen dos clases de Modbus en la industria: la American Standard Code for Information Interchange (ASCII) y la remote terminal unit (RTU), [5]. El Modbus RTU, el cual fue seleccionado para implementarse en este proyecto, requiere que cada byte se envíe como una cadena de ocho caracteres binarios enmarcada con un bit de inicio y un bit de parada, por lo que cada transmisión es de diez bits de longitud, la cual fue un factor determinante para su aplicabilidad en el proyecto.

Figura 1. Diagrama del proyecto para la red de comunicación desarrollada, [10].

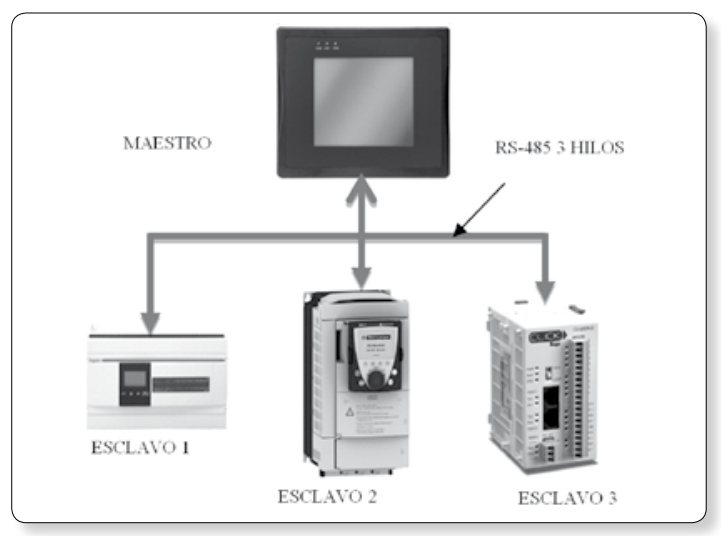

Figura 2. Capas de aplicación del protocolo Modbus en el modelo OSI [4].

\begin{tabular}{|c|c|c|}
\cline { 2 - 3 } & APLICACIÓN & MODBUS \\
\hline-6 & PRESENTACIÓN & \\
\hline-5 & SESIÓN & \\
\hline-4 & TRANSPORTE & \\
\hline-3 & RED & MAESTRO-ESCLAVO \\
\hline-2 & ENLACE DE DATOS & RS-485 \\
\hline 1 & FÍSICA & R \\
\hline
\end{tabular}

\section{Caracterización}

\section{$y$ construcción}

\section{experimental del módulo didáctico}

A continuación se describen las características principales de los dispositivos interconectados en la red de comunicación del módulo didáctico.

\subsection{HMI-5056N de la Maple Systems}

Es una interfaz gráfica sensible al tacto del operador o bien conocida como Human Machine Interface (HMI), facilita la supervisión de los operarios a plantas industriales intuitivamente. El comportamiento de estas interfaces es similar al comportamiento de los componentes discretos, por ejemplo al pulsar switch selector este toma la forma natural, igual que si fuera dispositivos discretos. Los operadores pueden hacer ajustes rápidamente de ayudas incluyendo setups de las máquinas, textos de diagnósticos, mantenimiento y alarmas, [7] [8]. Entre sus características principales se cuenta con un microprocesador de $400 \mathrm{Mhz}$ una memoria flash de $128 \mathrm{MB}$, para su conexión a la red cuenta con dos puertos físicos seriales que trabajan RS-232 y RS-485.

\subsection{PLC Twido TWDLCAE40DRF de Telemecanique}

El controlador lógico programable (PLC) Twido, cubre aplicaciones estándar de 10 a 264 entradas/salidas. Presenta un diseño compacto y su operación, según el fabricante, es sencilla y fiable. Permite comunicaciones Modbus [5]. El fabricante destaca su facilidad de integración, cableado y montaje, para la adaptación a necesidades concretas, ahorran tiempo y mejoran la fiabilidad. Se destina a instalaciones sencillas y máquinas compactas. Para su conexión a la red cuenta con un puerto serial minidim de 8 pines que trabaja conexión física RS-232 y RS-485. 


\subsection{Variador de frecuencia ALTIVAR 71}

El variador de frecuencia modelo Altivar 71 de la empresa Schneider Electric, permite ejecutar diferentes tipos de control a motores con una gran funcionalidad integrada. Está adaptado a los siguientes accionamientos: par y precisión de velocidad, dinámica elevada con control vectorial de flujo, gama de frecuencia ampliada para los motores de alta velocidad, puesta en paralelo de motores y accionamientos especiales gracias a la ley de tensión/frecuencia. Para su conexión a la red cuenta con un puertos RJ-45 que trabaja conexión física RS-232 y RS-485, [9].

\subsection{PLC Click Koyo c0-02dd2-d}

Es un controlador lógico programable (PLC) que cubre aplicaciones análogas y digitales, consta de dos canales de entrada y salidas analógicos de corriente (4-20 mA) y voltaje $(0-5 \mathrm{Vdc})$. La memoria es de 8.000 pasos de programa, además tiene tres puertos de comunicación, uno con la una única función de programación RS232C, un segundo puerto para comunicaciones seriales bajo una conexión física RS232C y finalmente un tercer puerto que fue utilizado en el módulo didáctico bajo la referencia RS485 Modbus.

\subsection{Construcción experimental del módulo didáctico}

Una vez realizada la caracterización y selección de los elementos necesarios para la ejecución del proyecto, es importante resaltar que la construcción del modulo involucró el desarrollo del proyecto a nivel de software y a nivel de hardware. A nivel de software, el desarrollo de las comunicaciones se programó en cada elemento de la red de forma independiente, teniendo en cuenta que la información entre los dispositivos debe estar en una continua transferencia basándose en direcciones lógicas y registros en cada esclavo coordinados dentro de la estructura de la automatización. Además se programó en cada equipo su nombre de red y su posición previamente establecida como lo muestra la figura 3 .

Figura 3. Configuración software de la red de comunicación en el módulo didáctico, [10].

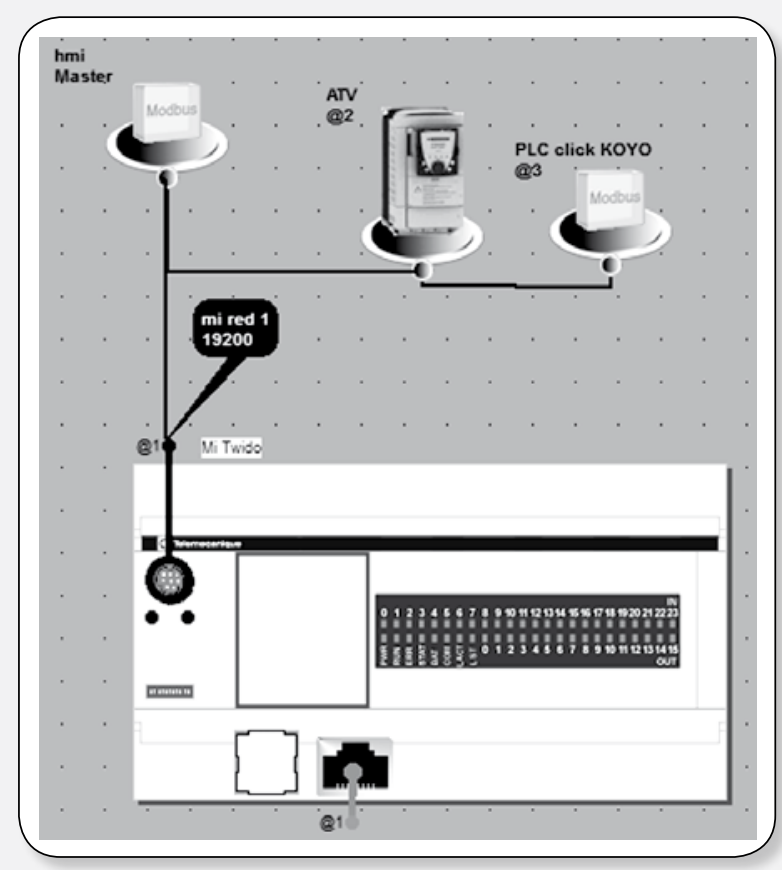

Para establecer los parámetros de comunicación se interconectaron físicamente los esclavos individualmente para comprobar la capacidad de cada dispositivo, se observó que el PLC Twido y el variador de frecuencia altivar 71 no presentaron ninguna respuesta a velocidades bajas de transferencia de datos específicamente a $9600 \mathrm{kbit} / \mathrm{s}$. Se estableció que para $19200 \mathrm{kbit} / \mathrm{s}$, todos los equipos soportaban correctamente la red de comunicación entre si, por lo tanto los parámetros de comunicación fueron establecidos de acuerdo con la siguiente tabla 1.

Tabla 1. Parámetros de comunicación.

\begin{tabular}{|c|c|}
\hline PARÁMETRO & VALOR \\
\hline Velocidad de trasmisión & 19200 baudios \\
\hline Bits de datos & 8 bits \\
\hline Paridad & Ninguna \\
\hline Bits de parada & 1 bits \\
\hline Tiempo de espera & 0,1 segundos \\
\hline Retraso de entrega & 1 milisegundo \\
\hline
\end{tabular}


La pantalla HMI 5056N que hace el papel de maestro en esta red de comunicaciones trabaja una comunicación half-duplex con cada uno de los elementos interconectados, se hizo necesario programar la pantalla HMI para que renvié dos veces los paquetes de información a sus esclavos de forma que no se presentará perdida de información.

A nivel de Hardware, fue necesario la construcción de una fuente de alimentación de $24 \mathrm{Vdc}$ para el suministro de energía del PLC Click y la pantalla HMI 5056N, los demás dispositivos (plc twido Telemecanique y el variador altivar 71) se alimentan directamente de la red de distribución.

El módulo consta de dos paneles frontales, el superior que esta formado por la etapa de protección eléctrica, la tierra del módulo, la fuente de alimentación de $24 \mathrm{Vdc}$ y el PLC Twido con sus 24 entradas y 16 salidas directamente conectadas a borneras para fácil acceso.

En las figuras 4 y 5 , se puede observar la etapa inicial de la construcción y el prototipo final del módulo identificándose los dispositivos que conforman la red de comunicaciones como lo son el variador de frecuencia Altivar 71, el PLC click koyo, el PLC twido y la pantalla HMI 5056N.

Figura 4. Etapa inicial en la construcción del prototipo del módulo didáctico.

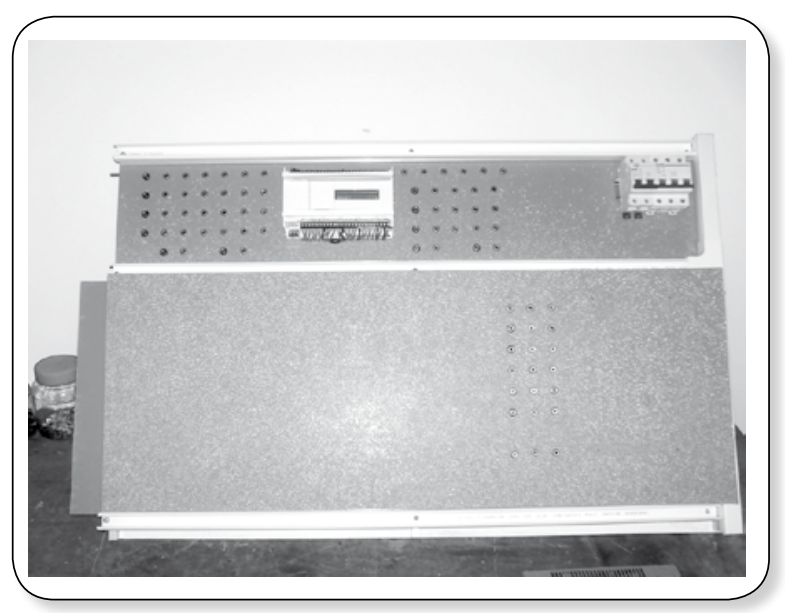

Figura 5. Vista final del módulo didáctico.

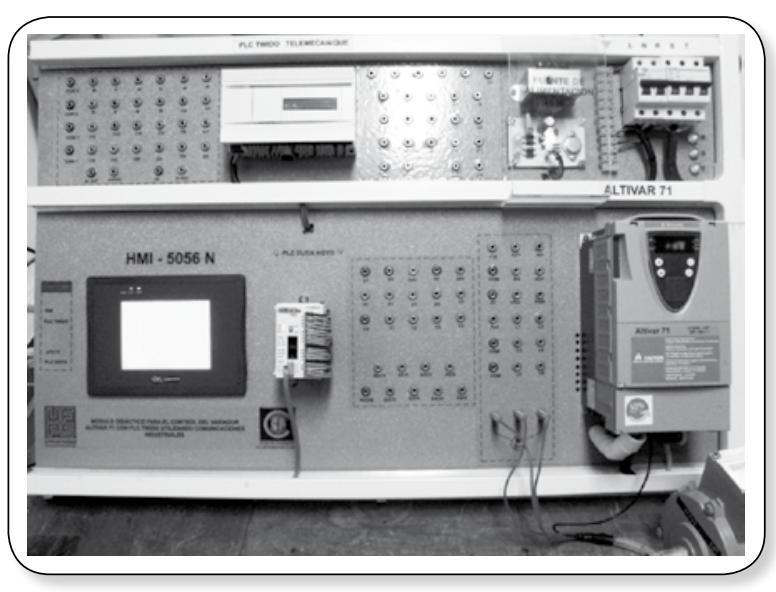

\section{Pruebas}

\section{experimentales.}

Las aplicaciones del módulo didáctico utilizando la pantalla HMI5056N están constituidas por uno o más ventanas dentro de las cuales se encuentran algunos objetos y sub-rutinas cuyas propiedades (color, posición, sensibilidad, etc.) pueden variar a las señales de control (activación por un sensor, un final de carrera, etc.). Otros objetos pueden ser modificados por el operador (pulsando el área programada) para suministrar ordenes a los PLCs desde la pantalla HMI a través de la red de comunicación industrial desarrollada.

Una de las pruebas experimentales es la activación del variador de frecuencia altivar 71 por medio de la red Modbus implementada. Se inicia a partir del acceso al registro de control (CMD) de la memoria interna del variador. Una vez realizado se permite a través de la red controlar la consigna de velocidad (LFRD) dada en revoluciones por minuto (RPM) y limitada por los valores previamente configurados en la pantalla. Esta pantalla, como puede ser observada en la figura 6, consta de un medidor gráfico tipo aguja que lee el valor de velocidad actual y lo marca en su escala previamente configurada y cuatro set bits que tienen como finalidad modificar parámetros específicos en el registro de control (CMD), reset (modifica el bit 3), marcha atrás 
(modifica el bit 2), marcha adelante (modifica el bit 1) y stop que lleva a cero todos los bits del registro CMD deteniendo por completo el funcionamiento del variador.

La figura 7 presenta la última ventana en la HMI5056N en comunicación con los demás dispositivos del módulo didáctico, dicha ventana permite la supervisión y monitorización de los parámetros del variador de frecuencia, como son los valores actuales de lectura visualizados directamente por medio de displays numéricos, a estos parámetros se les permite únicamente la opción de lectura, además si el usuario desea conocer el comportamiento de la variable a través del tiempo puede acceder a una ventana de gráfico. Además pulsando las teclas de función ubicadas a mano izquierda de cada parámetro, se permite la adquisición de datos en tiempo real trabajando con el data sampling, de forma que la transferencia de datos se ejecuta a través de la creación de una matriz dinámica en el tiempo.

Figura 6. Pantalla puesta en marcha modo on-line

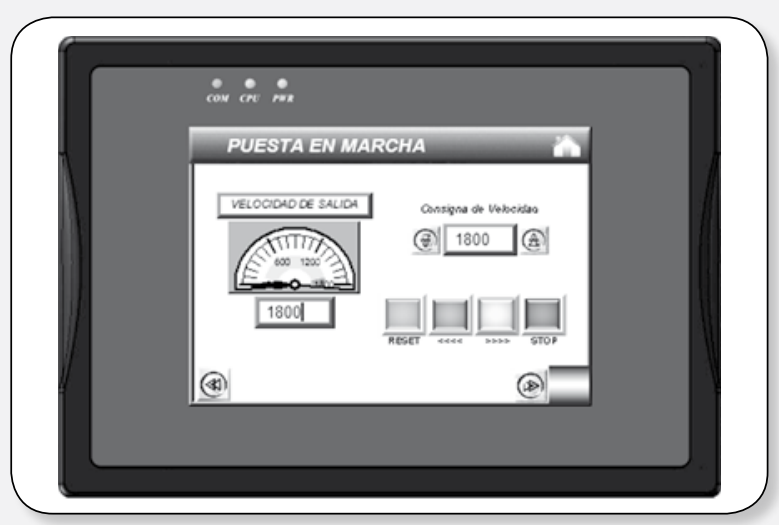

Figura 7. Pantalla supervisión en tiempo real, trend Display.

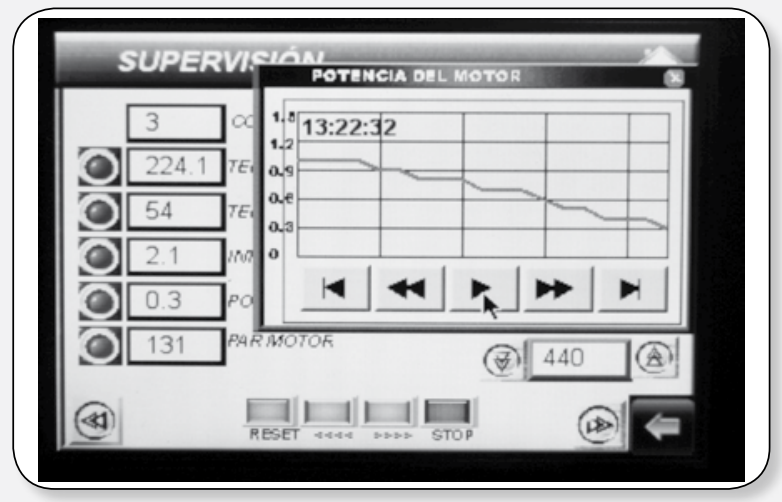

\section{Conclusiones}

ISSN 0122-820X

Se diseñó e implementó un módulo didáctico que permite establecer la comunicación entre controladores lógicos programables, actuadores y una interfaz gráfica que permite la monitorización de los elementos pertenecientes a la red de comunicación industrial.

Con la implementación del protocolo MODBUS en el módulo didáctico, se evidencia la facilidad y flexibilidad del uso de este protocolo. Ésta es quizás la principal razón de su alta difusión en entornos industriales. Se realizaron pruebas en las cuales fue visible la capacidad de operación en red e interoperabilidad del modulo, de forma que desde clientes Modbus de diferentes fabricantes accedan a registros y datos sobre los diversos elementos que conforman la red.

Para el manejo del módulo, se debe realizar una programación previa de los PLC's a utilizar, que se cumpla la verificación de la comunicación entre la pantalla HMI, el variador y los PLC's, por lo cual se da la posibilidad de trabajar con conexión ON LINE, cuya finalidad es comprobar el intercambio de registros, ya sean utilizados para salidas y entradas en tiempo real.

Las herramientas trabajadas en éste proyecto, permite a los estudiantes familiarizarse con la supervisión, control de procesos, la programación de autómatas programables y adaptación de tecnologías en el área de la automatización industrial. Además es importante resaltar entre los resultados académicos más importantes de este proyecto la realización de artículos de carácter científico y guías para desarrollar laboratorios utilizando este modulo didáctico por parte de la comunidad estudiantil.

Se pueden crear herramientas para laboratorios de electrónica y comunicaciones industriales invirtiendo aproximadamente un $40 \%$ menos de economía por debajo 
de los costos que representaría adquirir un equipo comercial de una casa fabricante con representación internacional.

Cuando se enlazan los elementos de la red con la pantalla Touch Screen, se debe configurar el formato y características de comunicación entre ellos, como la disponiblidad de los puertos de comunicación de la pantalla solo era uno a la vez (COM 1), fue necesario utilizar el driver Modbus RTU master, adaptando el variador y el PLC click $\mathrm{KOYO}$ a este formato. Es muy importante el concepto de este tipo de protocolo, para evitar posibles conflictos de comunicación.

Se evidencia cómo una interfaz gráfica permite supervisar una planta por medio de controladores o PLC's interconectados a través del protocolo Modbus, por ejemplo, que un sólo operario pueda atender remotamente diversos procesos simultáneamente desde un mismo puesto de trabajo. Además del monitoreo tradicional de variables es posible cambiar los parámetros operativos individuales de los controladores.

\section{Referencias bibliográficas}

[1] B. AKTAN, C. BOHUS, L. CROWL, AND M. SHOR, "Distance learning applied to control engineering laboratories," IEEE Transactions on Education, vol. 39, no. 3, pp. 320-326, Aug 1996.
[2] DIEGO J. SAGI, PABLO F. BLANCO, FRANCISCO J. DOMINGO. Estándares en tecnologías educativas. Universidad Complutense de Madrid, 2010. Disponible en internet: http:// www.cesfelipesegundo.com/revista/ Articulos2004/Articulo11.pdf.

[3] CETIS. "Learning Technology Standards: An Overview. CETIS (The center for educational technology interoperability standards)", 25 de
Noviembre de 2001. Disponible en internet: http://www.cetis.ac.uk/static/ standards.html.

[4] K. PAPASIDERIS, C. LANDRY, B. SUTTER AND A. WILSON "Environment Temperature Control Using Modbus and RS485 Communication Standards"Engineering Technology and Industrial Distribution Department Texas A\&M University Faculty Advisor: Dr. Joseph Morgan, D.E., P.E. January 10, 2009.

[5] VITOR C.L.D. Redes de Automação: Controlnet, Devicenet e Fieldbus, Controle \& Instrumentação. São Paulo, Publicações Valete, set 2000, p 68 - 74.

[6] URDA, DIEGO CARROZO. Protocolo Modbus. Santa fe de Bogotá: McGraw Hill, 2003, 348 p.

[7] MAPLE SYSTEMS. Manuales de funcionamiento, programación y operación de las Pantallas Touch screen HMI5056N de la maple systems, 2011. Disponible en Internet: http://www. maplesystems.com/

[8] NATALE F. Automação Industrial. Ed. Érica. 6” Edição. São Paulo, 2000, 256p.

[9] SCHNEIDER ELECTRIC ESPAÑA, S.A. - Manuales Altivar 71, Catalogo 2005. Disponible en Internet: http:// www.schneiderelectric.es

[10] MONDRAGON E. JORGE E. "Diseño e implementación de un sistema didáctico para el control del convertidor altivar 71 con plc Twido utilizando comunicaciones industriales" 2012. 198p Tesis. Universidad Francisco de Paula Santander, Colombia 